\title{
The Cancer-Testis Gene, NY-ESO-1, Is Expressed in Normal Fetal and Adult Testes and in Spermatocytic Seminomas and Testicular Carcinoma In Situ
}

\author{
Anne-Pascale Satie, Ewa Rajpert-De Meyts, Giulio C. Spagnoli, Sébastien Henno, \\ Laurence Olivo, Grete Krag Jacobsen, Nathalie Rioux-Leclercq, Bernard Jégou, and \\ Michel Samson
}

\begin{abstract}
GERM-INSERM U. 435 (A-PS, BJ, MS), Université de Rennes I, Campus de Beaulieu, Rennes, France; Department of Growth and Reproduction (ER-DM), Copenhagen University Hospital (Rigshospitalet), Copenhagen, Denmark; Departments of Surgery and Research (GCS), University of Basel, Switzerland; Service d'Anatomopathologie (SH, LO, NR-L), CHU Ponchaillou, Rennes, France; and Department of Pathology (GKJ), Gentofte University Hospital,
\end{abstract} Gentofte, Denmark

SUMMARY: Cancer/testis genes are potential targets for therapeutic genetic and immunologic approaches, and are highly expressed in a large variety of human cancers. However, they are not expressed in normal tissues, with the exception of the testis. The NY-ESO-1 gene is the most recently identified member of the cancer/testis family and its product is one of the most immunogenic tumor antigens. We used immunohistochemistry to investigate the expression of NY-ESO-1 in healthy human prenatal and adult testes and in 59 human testicular tumors of different subtypes. We found that NY-ESO-1 was expressed from 18 weeks until birth in human fetal testes. In the adult testis, NY-ESO-1 was strongly expressed in spermatogonia and in primary spermatocytes, but not in post-meiotic cells or in testicular somatic cells. NY-ESO-1 was not expressed in the Sertoli cells, Leydig cells, classical seminomas, or nonseminomatous germ cells in the 59 testicular tumors. In contrast, NY-ESO-1 was expressed both in carcinomas in situ, which are the earliest stage of testicular tumors ( 7 of 15 cases), and in spermatocytic seminomas, which are believed to be derived from spermatogonia or primary spermatocytes (8 of 16 cases). We conclude that NY-ESO-1 is a marker that can be used to follow the early progression of testicular tumorigenesis when the tumors present a similar pattern of expression to the cells from which they originated, although the later tumors cease to express NY-ESO-1. (Lab Invest 2002, 82:775-780)

$T$ esticular cancer is the most common form of cancer in men aged between 15 and 40 years. Its occurrence has been reported to have increased in a number of industrialized western countries (Adami et al, 1994; Moller, 1993; Osterlind, 1986). The etiology of testicular cancer is still very poorly understood. However, it is generally acknowledged that the carcinogenic process may be initiated before birth (Depue et al, 1983; Henderson et al, 1979). This highlights the importance of identifying specific testicular markers for each type of testicular tumor and for the early stages of neoplastic transformation. We aimed to identify such early markers by focusing on the members of the cancer/testis (CT) family as they are expressed both in a large variety of

\section{DOI: 10.1097/01.LAB.0000017169.26718.5F}

Received February 15, 2002

This work has been supported by INSERM, the Ministère de l'Education Nationale de la Recherche et de la Technologie, the Fondation pour la Recherche Médicale, the Association pour la Recherche sur le Cancer, the Ligue nationale contre le cancer and the Région Bretagne.

Address reprint requests to: Dr. Michel Samson, Université de Rennes I, GERM-INSERM U. 435, Campus de Beaulieu, 35042 Rennes cedex, Bretagne, France.E-mail:michel.samson@rennes.inserm.fr cancers and in the testis, but not in other organs. We recently demonstrated that MAGE-A4, a member of the CT family, is expressed in human testicular germ cells throughout development. MAGE-A4 is expressed in classical seminomas and in carcinomas in situ (CIS), but not in nonseminomas; thus it is possible to distinguish between the different types of testicular tumor (Aubry et al, 2001). Here, we focused on NY-ESO-1, which was recently characterized and shown to be expressed in normal testes (Jungbluth et al, 2001). Its product is one of the most immunogenic tumor antigens defined to date in the CT family (Jager et al, 2000). We also analyzed the expression of NY-ESO-1 in fetal and adult testes and in a large variety of different testicular tumors, including classical seminomas, CIS, nonseminomatous germ cell tumors (NSGCT), spermatocytic seminomas, and nongerm-cell tumors derived from Leydig and Sertoli cells.

\section{Results}

\section{Western Blot Analysis}

Six monoclonal antibodies (mAbs), namely B9-8, D838, B4-2, D1-13, D8-10, and B10-3, raised against the recombinant NY-ESO-1 fusion protein (Schultz-Thater 
et al, 2000), were tested on human testicular extracts (T) and the recombinant NY-ESO-1 protein (r). B9-8, D8-38, B4-2, D8-10, and B10-3 recognized both the recombinant and native proteins. However, in our conditions, D1-13 failed to recognize the target proteins. When the recombinant protein was used, a protein with an apparent molecular weight of $40 \mathrm{kDa}$ was revealed, whereas a band of $22 \mathrm{kDa}$ was detected in the testis extracts (Fig. 1).

\section{Immunohistochemical Analysis of the Expression of NY-ESO-1 in the Adult Testis}

D8-38 was selected for the immunohistochemical experiments because it presented a strong reactivity with the testicular extracts (Fig. 1). No staining was observed with the control mAb in the normal testis (Fig. 2A), but strong and specific staining was observed within the seminiferous tubules (Fig. 2B). The intensely labeled cells were identified as spermatogonia (Fig. 2C) and primary spermatocytes (Fig. 2D). This staining was localized in the cytoplasm. Spermatids (post-meiotic cells) and Sertoli cells (somatic cells) were consistently negative. Furthermore, no staining was observed in the seminiferous tubules from patients with Sertoli-cell-only syndrome (Fig. 2E).

\section{Immunohistochemical Localization of NY-ESO-1 in the Fetal Testis at Different Stages of Development}

Paraffin-embedded testis sections from 12-, 13-, 14-, 16-, 18-, 19-, 22-, 24-, 26-, 28-, 32-, 34-, and 40week-old human fetuses were labeled with the D8-38 antibody to investigate the localization of NY-ESO-1 at different developmental stages. No NY-ESO-1 staining was observed in the embryonic gonad before 16 weeks (Fig. 3A), but a few NY-ESO-1-positive cells were seen in the gonads of the 18-week-old fetus (Fig. $3 \mathrm{~B})$. The number of stained cells increased after 18 weeks and was maximal in the 40-week-old fetal gonad (Fig. 3C). At all stages studied, the NY-ESO-1positive cells were cytoplasmic, strictly located in the tubules, and presented the morphologic characteristics of gonocytes (Fig. 3C insert), the precursors of spermatogonia.

\section{NY-ESO-1 Expression in Testicular Tumors}

We used immunohistochemistry to investigate the expression of NY-ESO-1 in 59 testicular tumors using

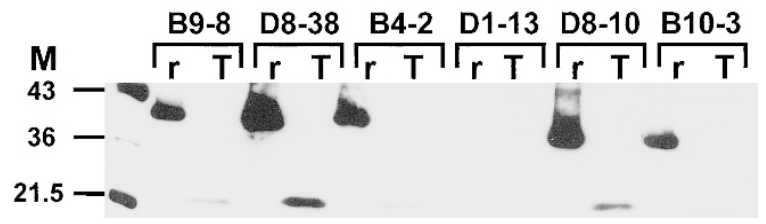

Figure 1.

Western blot analysis of recombinant NY-ESO-1 $(r)$ and a protein extract from human testicles $(T)$ using different monoclonal antibodies (1:40) raised against NY-ESO-1. The study was performed using $10 \mathrm{ng}$ of recombinant NY-ESO-1 protein $(r)$ or $40 \mu \mathrm{g}$ of protein extracted from whole human testes. The reactions were revealed using horseradish peroxidase conjugated to an antibody. $M$ : molecular mass markers (in $\mathrm{kDa}$ ). the D8-38 antibody. As expected, no staining was observed in somatic tumors (1 Sertoli cell and 2 Leydig cell tumors; data not shown). Surprisingly, no reaction was detected in the 12 classical seminomas (Fig. 4A) or in the 12 NSGCT (Fig. 4B, Table 1). In contrast, specific immunostaining was observed in 7 of $15 \mathrm{CIS}$ specimens screened (Fig. 4E: NY-ESO-1-positive CIS and Fig. 4G: NY-ESO-1-negative CIS). To confirm that CIS was present in all seminiferous tubules, including those that were negative for NY-ESO-1, we used an antibody against placental-like alkaline phosphatase (PLAP), which is routinely used to identify CIS cells (Giwercman et al, 1991). We showed that all of the CIS samples were PLAP-positive (Fig. 4, F and H). Furthermore, 8 of 16 spermatocytic seminomas tested NYESO-1-positive (Fig. 4C: NY-ESO-1-positive spermatocytic seminoma and Fig. 4D: NY-ESO-1-negative spermatocytic seminoma). The cytoplasm of cancer cells was also stained in a similar way to the germ cells from normal testes.

\section{Discussion}

The so-called CT genes are attractive targets for antigen-specific cancer therapy. A strategy based on spontaneous antibody responses to tumor associated antigens (SEREX) was recently used to identify a new CT antigen, named NY-ESO-1. This antigen is one of the most immunogenic tumor antigens defined to date, because it induces spontaneous humoral and CD8 + T cell responses against NY-ESO-1 in $40 \%$ to $50 \%$ of patients with advanced NY-ESO-1-expressing tumors (Jager et al, 2000). More recently, Kurashige et al (2001) showed that there is a positive correlation between the expression of NY-ESO-1 and the immunogenicity associated with the tumor grade in transitional cell carcinoma (Kurashige et al, 2001) and in melanoma (Goydos et al, 2001). These data suggest that NY-ESO-1 is a marker for the most advanced cancerous stages. Here, we used a number of mAbs directed against NY-ESO-1 (Schultz-Thater et al, 2000) to investigate the expression of NY-ESO-1 in the human testis at different developmental stages, and in various types of testicular tumors. As previously reported, the NY-ESO-1 mAbs specifically recognized the recombinant NY-ESO-1 protein (Schultz-Thater et al, 2000) but Western blotting showed that they also recognized the natural protein in testicular extracts. Our results for normal germ cells confirmed those of Jungbluth et al (2001), who showed that the expression of NY-ESO-1 was localized to spermatogonia in the cells. Moreover, our investigations revealed that NY-ESO-1 was strongly expressed in pachytene spermatocytes, but not in post-meiotic germ cells. As with MAGE-A4 (Aubry et al, 2001), the NY-ESO-1 gene was no longer expressed by the time of the second meiotic division. The NY-ESO-1 labeling was strictly limited to germ cells, as demonstrated by the absence of signal in the somatic cells within the normal testis with complete spermatogenesis, and in Sertoli-cell-only tubules. We studied the expression and localization of NY-ESO-1 at different developmental stages. NY- 


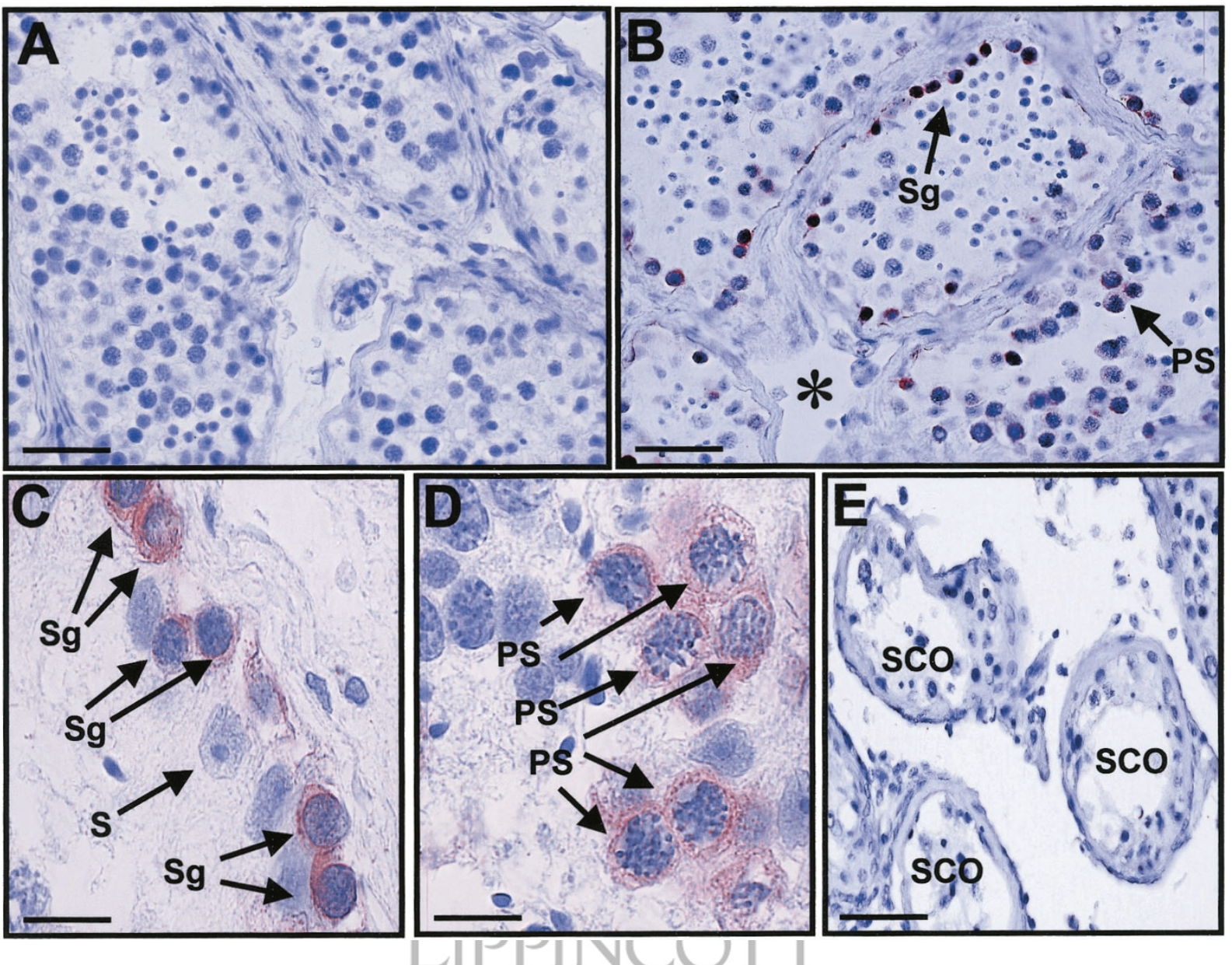

Figure 2.

Immunolocalization of NY-ESO-1 in the adult human testis. All tissues were fixed in paraformaldehyde. NY-ESO-1 was located using the monoclonal antibody D3-38 and an avidin-biotin peroxidase complex. The testis sections were either obtained from patients with no testicular diseases ( $A$ and $B$ ) or from patients suffering from Sertoli-cell-only (SCO) syndrome (E). These sections were incubated with the anti-NY-ESO-1 antibody (B and E) or control lgG1 (A); scale bar $=20 \mu \mathrm{m}$. A specific NY-ES0-1 signal was located within the seminiferous tubules. The interstitial tissue ${ }^{*}$ ) was consistently NY-ESO-1-negative (B). The positive cells were identified as being spermatogonia $(S g)(C)$ and pachytene spermatocytes $(P S)(D)$; scale bar $=100 \mu \mathrm{m}$. Sertoli cells $(S)$ were not labeled. None of the cell types in the $S C O$ testis were NY-ESO-1-positive (E).

ESO-1 was expressed from 18 weeks on in the spermatogonia precursors, gonocytes. A similar expression pattern was reported for MAGE-A4 (Aubry et al, 2001). Interestingly, the SSX protein (synovial sarcoma on $\mathrm{X}$ chromosome) is expressed in spermatogonia, primary spermatocytes, and gonocytes from at least the 17th week of development on (Stoop et al, 2001). This suggests that this stage is crucial in the production of several proteins involved in the development of the gonad.

The demonstration that NY-ESO-1 is expressed in gonocytes suggests that this CT can be used as a marker to follow testicular carcinogenesis. Indeed, NY-ESO-1 was specifically expressed in 7 of $15 \mathrm{CIS}$ and in 8 of 16 spermatocytic seminomas studied. CIS are considered to be gonocytes that were misprogrammed and are the earliest cellular stages of seminomas. Spermatocytic seminomas seem to have derived from spermatogonia and/or spermatocytes. In contrast, NY-ESO-1 was not expressed in somatic cell tumors (Sertoli and Leydig), in nonseminomatous germ cell tumors, or in seminomas. However, the
PCR-based approach used by Yuasa et al (2001) found that NY-ESO-1 was expressed in some seminomas. This discrepancy could result from the contamination of the cancerous testicular tissues used by Yuasa et al (2001) with the normal testicular tissue that always surrounds the tumor and which contains spermatogonia and primary spermatocytes, which express NY-ESO-1.

In our conditions, NY-ESO-1 was only expressed in $47 \%$ of CIS, whereas PLAP was in all CIS. This observation reveals the molecular heterogeneity of CIS. It is possible that the seminomas and NSGCT that did not express NY-ESO-1 had lost NY-ESO-1 during tumor progression. Consequently, the CIS cells that were positive for NY-ESO-1 may have retained the gonocyte-like phenotype, whereas those that were negative for NY-ESO1 could be pre-invasive precursors that are already partly differentiated toward seminomas or NSGCT. Spermatocytic seminomas, which are thought to be derived from spermatogonia/spermatocytes (Stoop et al, 2001), expressed NY-ESO-1, as did the spermatogonia and primary spermatocytes 


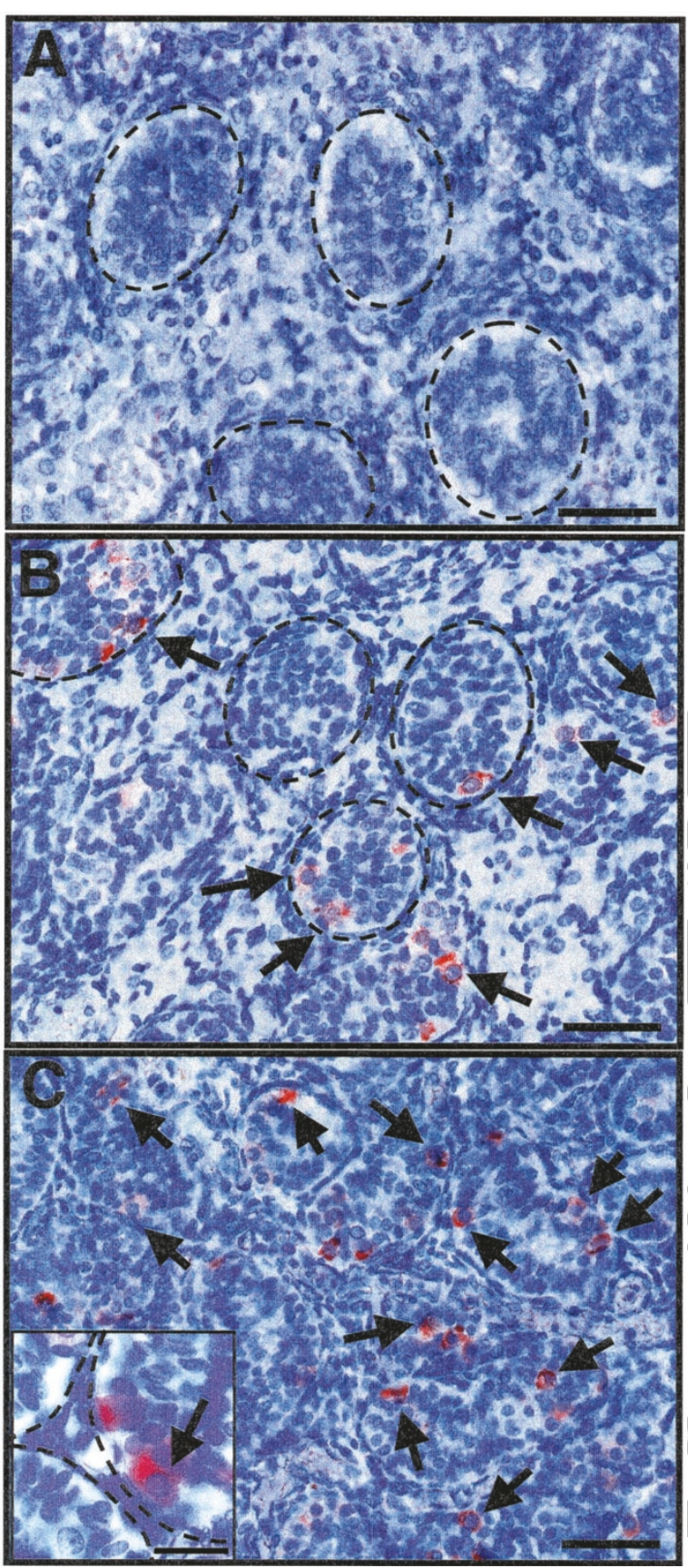

Figure 3.

Immunolocalization of NY-ESO-1 in human fetal male gonad. Sections of human 16-week-old (A), 18-week-old (B), and 40-week-old fetal gonads (C) were incubated with the D3-38 antibody. The dotted lines indicate the sex-cords in A, B, and C. Arrows show positively labeled cytoplasmic gonocytes (B and $C$ ). Scale bars $=20 \mu \mathrm{m}$. The scale bar in the insert $=50$ $\mu \mathrm{m}$.

in normal tissue. A heterogeneous pattern of NYESO-1 expression in spermatocytic seminoma might indicate the molecular heterogeneity of this tumor, perhaps reflecting different stages of the tumor progression. Interestingly, although NY-ESO-1 and MAGE-A4 belong to the same CT family and share similar characteristics, they also present specific features. Whereas most (87\%) CIS samples were MAGEA4-positive (Aubry et al, 2001), only $47 \%$ were NY-
ESO-1 positive. Similarly, although nearly all of the spermatocytic seminoma samples studied were MAGE-A4-positive (Rajpert-De Meyts et al, 2002), only $50 \%$ were NY-ESO-1-positive. Thus, a better understanding of the expression patterns of the different CT members should allow us to precisely classify the different types of the CIS-derived testicular tumors and their developmental stages.

In conclusion, we have shown that NY-ESO-1 is specifically expressed in some CIS and in spermatocytic seminomas but not in seminomas, NSGCT, or somatic cell tumors (derived from Sertoli and Leydig cells). NY-ESO-1 can be used as a marker to improve our understanding of the cellular origin of malignant cells and the progression of the tumors.

\section{Materials and Methods}

\section{Tissue Specimens and Clinicopathologic Data}

Fifty-nine specimens of testicular tumors and tissues were obtained from patients with testicular cancer who underwent surgery at one of several hospitals in France, Denmark, and Sweden. The series included 12 seminoma specimens, 13 NSGCT specimens with various histologic components, $15 \mathrm{CIS}$ specimens, 16 spermatocytic seminoma specimens, 1 Sertoli cell tumor, and 2 Leydig cell tumors (Table 1). Three normal testis specimens were used as positive controls. Sections of fixed gonads from 12- to 40-weekold fetuses were obtained from the pathology department of Ponchaillou Hospital, France. These tissue specimens were isolated after induced or spontaneous abortions when routine autopsies found no signs of disease or developmental disorders. The study was approved by local ethical committees.

\section{Western Blot Analysis of NY-ESO-1}

Proteins were subjected to SDS-PAGE on $12.5 \%$ polyacrylamide gels. We transferred $40 \mu \mathrm{g}$ of crude protein extracts from human testes or $10 \mathrm{ng}$ of recombinant NY-ESO-1 protein onto Immunobilon- $P^{S Q}$ (PVDF) membranes (Millipore, Saint Quentin en Yvelines, France) as described by Towbin et al (1992). The membranes were blocked overnight at $4^{\circ} \mathrm{C}$ in $0.02 \mathrm{M}$ Tris buffered saline (TBS) supplemented with $3 \%$ BSA. After washing with TBS, the membranes were incubated for 2 hours at room temperature with mAbs directed against NY-ESO-1 (working dilution, 1:40) in TBS supplemented with $0.1 \%$ Tween and $1 \%$ BSA. After washing in TBS-Tween $0.1 \%$, the membranes were incubated with a 1/5,000 dilution of horseradish peroxidase conjugated anti-mouse antibody (Amersham Pharmacia Biotech, Les Ulis, France). After several washes in TBS-Tween $0.1 \%$, antibody binding was detected by use of ECL (Amersham Pharmacia Biotech). Kodak Biomax films were exposed to the blots for 15 minutes.

\section{Immunohistochemical Localization of NY-ESO-1}

Tissue sections were deparaffinized and rehydrated. They were then incubated for 5 minutes in $3 \% \mathrm{H}_{2} \mathrm{O}_{2}$ to 

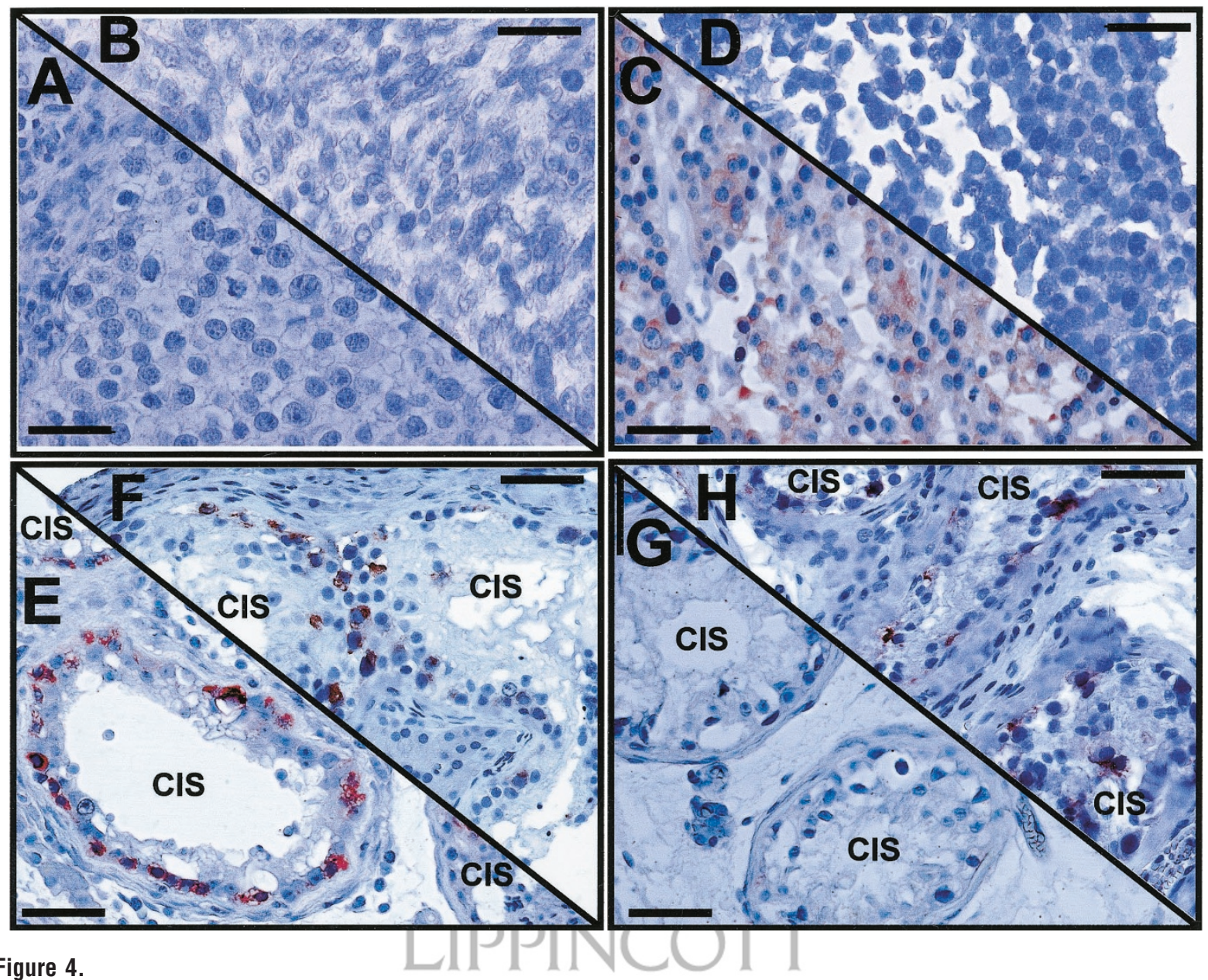

Immunolocalization of NY-ESO-1 in a range of testicular tumors. Sections of human testicular tumors were fixed in paraformaldehyde and incubated with either an anti-NY-ESO-1 (D3-38) antibody (A to E, and G) or an änti-placental-like alkaline phosphatase antibody ( $\mathrm{F}$ and $\mathrm{H})$. Seminomas ( $\bar{A}$ ), nonseminomatous germ cell tumors (B), spermatocytic seminomas (D), and tubules presenting CIS (G) were negative for NY-ESO-1. Spermatocytic seminoma (C) and some cancer-in-situ $(\mathrm{CIS})$-containing tubules (E) were positive for NY-ESO-1. Tubules presenting cancer-in-situ were positive for placental-like alkaline phosphatase (F and G). Scale bars $=100 \mu \mathrm{m}$.

Table 1. Expression of NY-ESO-1 in Testicular Tumors

\begin{tabular}{lrc}
\hline Type of testicular tumor & $n$ & Positive staining \\
\hline Seminoma & 17 & 0 \\
NSGCT & 12 & 0 \\
CIS & 15 & 7 \\
Spermatocytoma & 16 & 8 \\
Sertoli cell tumor & 1 & 0 \\
Leydig cell tumor & 2 & 0 \\
\hline
\end{tabular}

block endogenous peroxidase activity, rinsed in TBS, and incubated twice for 10 minutes in TBS supplemented with 1\% BSA to block the nonspecific sites. The tissue sections were incubated overnight at $4^{\circ} \mathrm{C}$ with either a 1:50 dilution (in 1\% BSA) of the anti-NYESO-1 antibody (mouse monoclonal antibody D8-38) or with $1 \mu \mathrm{g} / \mathrm{ml}$ mouse IgG1 (DAKO, Glostrup, Denmark) as a negative control. All subsequent steps were performed at room temperature. After several washes with TBS, the sections were incubated for 2 hours with a biotinylated rabbit anti-mouse IgG at a dilution of 1:500, washed again with TBS, and then incubated with a 1:500 dilution of peroxidase-conjugated streptavidin (DAKO) for 30 minutes. After a final rinse with TBS, the sections were incubated with aminoethyl-carbazole substrate (DAKO) for 30 minutes to reveal the specific red staining. The nuclei were counterstained with Hemalun Masson solution. The sections were photographed using an Olympus AX60TF microscope with monochromatic objectives (Olympus, Paris, France), coupled to a digital macro camera (Kigamo, Metis, France).

\section{References}

Adami HO, Bergstrom R, Mohner M, Zatonski W, Storm H, Ekbom A, Tretli S, Teppo L, Ziegler H, Rahu M, Gurevicius R, and Stengrevics A (1994). Testicular cancer in nine northern European countries. Int J Cancer 59:33-38.

Aubry F, Satie AP, Rioux-Leclercq N, Rajpert-De Meyts E, Spagnoli GC, Chomez P, De Backer O, Jegou B, and Samson M (2001). MAGE-A4, a germ cell specific marker, is expressed differentially in testicular tumors. Cancer 92: 2778-2785. 
Depue RH, Pike MC, and Henderson BE (1983). Estrogen exposure during gestation and risk of testicular cancer. J Natl Cancer Inst 71:1151-1155.

Giwercman A, Cantell L, and Marks A (1991). Placental-like alkaline phosphatase as a marker of carcinoma-in-situ of the testis: Comparison with monoclonal antibodies M2A and 43-9F. APMIS 99:586-594.

Goydos JS, Patel M, and Shih W (2001). Ny-eso-1 and ctp11 expression may correlate with stage of progression in melanoma. J Surg Res 98:76-80.

Henderson BE, Benton B, Jing J, Yu MC, and Pike MC (1979). Risk factors for cancer of the testis in young men. Int J Cancer 23:598-602.

Jager E, Gnjatic S, Nagata Y, Stockert E, Jager D, Karbach J, Neumann A, Rieckenberg J, Chen YT, Ritter G, Hoffman E, Arand M, Old LJ, and Knuth A (2000). Induction of primary NY-ESO-1 immunity: CD8+ T lymphocyte and antibody responses in peptide-vaccinated patients with NY-ESO-1+ cancers. Proc Natl Acad Sci USA 97:12198-12203.

Jungbluth AA, Chen YT, Stockert E, Busam KJ, Kolb D, Iversen K, Coplan K, Williamson B, Altorki N, and Old LJ (2001). Immunohistochemical analysis of NY-ESO-1 antigen expression in normal and malignant human tissues. Int $\mathrm{J}$ Cancer 92:856-860.

Kurashige T, Noguchi Y, Saika T, Ono T, Nagata $Y$, Jungbluth A, Ritter G, Chen YT, Stockert E, Tsushima T, Kumon H, Old LJ, and Nakayama E (2001). NY-ESO-1 expression and immunogenicity associated with transitional cell carcinoma: Correlation with tumor grade. Cancer Res 61:4671-4674.

Moller H (1993). Clues to the aetiology of testicular germ cell tumours from descriptive epidemiology. Eur Urol 23:8-13; discussion 14-15.
Osterlind A (1986). Diverging trends in incidence and mortality of testicular cancer in Denmark, 1943-1982. Br J Cancer 53:501-505.

Rajpert-De Meyts E, Jacobsen GK, Aubry F, Bartkova J, Samson M, Bartek J, and Skakkebæk NE (2002). Expression pattern of selected onco-fetal antigens and cell-cycle regulators confirms the pre-meiotic origin of spermatocytic seminoma. Submitted.

Schultz-Thater E, Noppen C, Gudat F, Durmuller U, Zajac P, Kocher T, Heberer M, and Spagnoli GC (2000). NY-ESO-1 tumour associated antigen is a cytoplasmic protein detectable by specific monoclonal antibodies in cell lines and clinical specimens. Br J Cancer 83:204-208.

Stoop H, van Gurp R, de Krijger R, Geurts van Kessel A, Koberle B, Oosterhuis W, and Looijenga L (2001). Reactivity of germ cell maturation stage-specific markers in spermatocytic seminoma: Diagnostic and etiologic implications. Lab Invest 81:919-928.

Towbin H, Staehelin T, and Gordon J (1992). Electrophoretic transfer of proteins from polyacrylamide gels to nitrocellulose sheets: Procedure and some applications. 1979 Biotechnology 24:145-149.

Yuasa T, Okamoto K, Kawakami T, Mishina M, Ogawa O, and Okada Y (2001). Expression patterns of cancer testis antigens in testicular germ cell tumors and adjacent testicular tissue. J Urol 165:1790-1794.

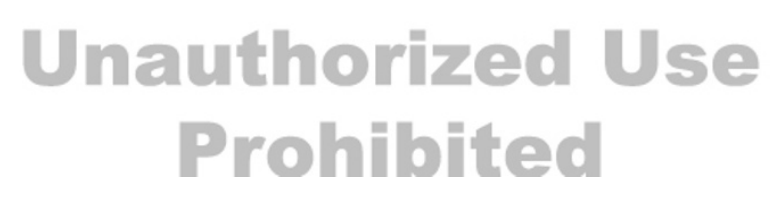

